JOURNAL OF

APPLIED

CRYSTALLOGRAPHY

ISSN 1600-5767

Received 13 June 2020

Accepted 27 August 2020

Edited by J. M. García-Ruiz, Instituto Andaluz de Ciencias de la Tierra, Granada, Spain

Keywords: periodicity; aperiodicity; 3D printing; hands-on learning.

Supporting information: this article has supporting information at journals.iucr.org/j

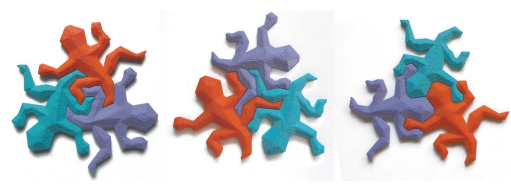

C 2020 International Union of Crystallography

\section{Teaching periodicity and aperiodicity using 3D-printed tiles and polyhedra}

\author{
Lluís Casas* \\ Crystallography and Mineralogy Unit, Geology Department, Universitat Autònoma de Barcelona, Edifici C, Campus de la \\ UAB, Bellaterra, Barcelona 08193, Spain. *Correspondence e-mail: Iluis.casas@uab.cat
}

Unit cell and periodicity are key concepts in crystallography and classically were thought to be inherent properties of ordered media like crystals. Aperiodic crystals (including quasicrystals) forced a change of paradigm, affecting the actual definition of a crystal. However, aperiodicity is usually not taught in crystallography undergraduate courses. The emergence of low-cost 3D-printing technologies makes it possible to tackle hands-on learning of the commonly taught crystallography concepts related to periodicity and to introduce in an uncomplicated manner aperiodic crystals and their related concepts that usually are skipped. In this paper, several examples of the use of 3D printing are shown, including 2D and 3D examples of periodic and aperiodic ordered media; these are particularly useful to understand both conventional periodic crystals and quasicrystals. The STL files of the presented models are made available with the paper.

\section{Introduction}

Crystallography started in the 17th century as the science for the study of the external shapes of crystals. Systematic study of crystal shapes led to enunciation of the law of the constancy of interfacial angles and soon it was argued that crystals must consist of ordered arrangements of atoms or molecules in a lattice (space-lattice hypothesis). In the early 20th century, soon after the discovery of X-rays (1895), diffraction of an $\mathrm{X}$-ray beam by a crystal contributed simultaneously to revealing the nature of X-rays (electromagnetic waves) and to confirming the space-lattice hypothesis. This led to the development of modern $\mathrm{X}$-ray diffraction techniques and $\mathrm{X}$-ray crystallography. Diffraction techniques are widely used to identify and ascertain the crystal structures of all kinds of solid substances, from organic to inorganic solids, pharmaceuticals, biological substances such as proteins and viruses etc. Effective application of X-ray diffraction techniques in geology, solid-state chemistry and materials science requires a basic understanding of crystallography.

From the academic point of view, crystallography is often present in secondary school chemistry courses through the study of crystal growth and sometimes as an accessory part within earth sciences courses (as part of mineralogy). Crystallography is sometimes present in innovative teaching tasks, including project-based learning, through experiments and competitions of crystal growth. The aesthetic qualities of crystals, as well as the symmetry of their idealized representations and that of lattices, can grab the attention of students and motivate them to pursue further studies of the subject. Crystallography and X-ray techniques are present in geology graduate programmes (Hluchy, 1999) as an independent topic or as a significant part of mineralogy courses. 
Moreover, many physics, chemistry (Fanwick, 2007; Pett, 2010) and materials science graduate programmes also include introductory notes on crystallography (Borchardt-Ott, 2012). Some undergraduate institutions have adapted the pedagogy of hands-on research to crystallography with the use of singlecrystal desktop instruments (Crundwell et al., 1999), and many other affordable virtual (Arribas et al., 2014) and tangible resources are available as educational supporting material for a number of important concepts in crystallography (Gražulis et al., 2015, and references therein). As many of these concepts require capacity for abstraction and spatial vision, many educators are taking advantage of the rise of 3D-printing technologies to develop interactive haptic environments for education. Of the 47 peer-reviewed articles listed in a recent systematic review of 3D printing in chemistry education (Pernaa \& Wiedmer, 2019), many deal with the production of physical models of molecular and crystal structures.

Periodicity and unit cell are key concepts in crystallography and they were fundamental in the classical definition of a crystal (i.e. 'a periodic array of atoms or group of atoms packed along the three space dimensions'; Authier \& Chapuis, 2017). Periodicity of common crystals implies that they can be generated by a list of independent finite translations, and this results in a finite number of allowed rotational symmetries (only two-, three-, four- and sixfold rotational symmetry is permitted) (Sharma, 1983); therefore the number of symmetry groups is also limited (e.g. 32 for 3D crystallographic pointgroup symmetry). These restrictions are part of the well known crystallographic restriction theorem, closely related to Haüy's crystallographic 'law of rationality' (Coxeter, 1973).

However, in the 1980s, the discovery of quasicrystals (Shechtman et al., 1984) broke the established paradigm as these intermetallic compounds can exhibit forbidden symmetries such as five-, eight-, ten- or 12-fold symmetry axes (Kortan et al., 1989; Fisher et al., 1999) and accordingly they exhibit diffraction patterns with Bragg peaks arranged with the same forbidden symmetries (Tsai \& Cui, 2015; Maciá Barber, 2019). Quasicrystals are a type of 'aperiodic crystals' along with incommensurately modulated phases (IMPs) and composite crystals (CCs). However IMPs and CCs can be seen, respectively, as incommensurate modulations and intergrowths of $d$-dimensional periodic structures (Janssen $e t$ al., 2018). The possibility of exhibiting non-crystallographic rotational diffraction symmetry is restricted to quasicrystals because the other types of aperiodic crystals have periodic average structures. By 1992, the IUCr had replaced the classical definition of a crystal by 'any solid with an essentially discrete diffraction diagram' (Senechal, 2015) (i.e. sharp Bragg peaks and possibly a weak continuous background). For an updated and more detailed definition of a crystal and a discussion of it and some related concepts (such as order and non-crystalline order) readers are referred to the article by Grimm (2015).

Crystals and lattice periodicity are often taught in crystallography undergraduate courses without mention of aperiodic crystals, or they are mentioned just as a very particular subject. Tangible models of periodicity for teaching in classrooms are sometimes used. The unit cell, along with its atomic content, can be used to build a potentially infinite model of a crystal. 3D-printed models of unit cells have been suggested as educational materials produced from standard crystallography files (Chen et al., 2014; Rodenbough et al., 2015), and some crystal structure modelling software such as Mercury (Macrae et al., 2020) allows users to generate directly 3D-printable models. In contrast, tangible materials for teaching aperiodicity of quasicrystals are scarce (Rossi et al., 2020). In the present paper several examples of the use of 3D printing are offered along with the corresponding STL files. These are 2D and $3 \mathrm{D}$ examples of periodic and aperiodic ordered media, particularly useful to aid understanding of both conventional periodic crystals and quasicrystals.

Printable STL files were obtained from designs of tiles, polyhedra and unit-cell models that were made using freely downloadable Google SketchUp software. The files were then printed using acrylonitrile butadiene styrene (ABS) filament on a UP Plus $3 \mathrm{D}$ printer. The pieces were generally spray painted and occasionally hand painted using acrylic paint. In the following sections periodic and aperiodic lattices are treated separately. For both types of lattices, simpler 2D models are shown first and then more complex 3D ones.

\section{Periodic lattices}

\subsection{Theoretical background}

There are many fundamental concepts derived or attached to the definition of periodic crystals, and many are common to both 2D and 3D lattices. Some of the relevant concepts that can be explained using the presented models of periodic lattices are commented on very briefly below.

Restriction theorem. The application of symmetry operations contained in a periodic lattice must leave the set of lattice points, as well as the atomic content of the lattice, unchanged. As a result of this, rotational symmetry of periodic media is limited to one-, two-, three-, four- and sixfold axes (or centres in two dimensions) as established by the crystallographic restriction theorem (Scherrer, 1946).

Unit cell. The internal order of periodic crystals can be illustrated in many ways. The crystal lattice is often represented by a regular grid of equivalent points, and a set of linearly independent vectors is selected to describe all possible translations between equivalent points; these are used to define the unit cell. The choice of the vectors, and therefore the unit cell itself, is not unique. Generally, the unit cell is selected to be as small as possible but keeping the symmetry of the lattice (and its atomic content). The unit cell can be shifted by all combinations of lattice vectors filling the space without gaps or overlap.

Asymmetric unit. Within the unit cell, the asymmetric unit can be defined as a portion without internal symmetry (considering the atomic content) from which, by application of the symmetry operations of the space or plane group, the whole unit cell and ultimately the whole space can be filled. The term 'asymmetric unit' does not imply that the region has 
an asymmetric shape and, regarding their regular limits, the only constraints are defined by mirror planes and rotation axes. Consequently, pieces bearing curved faces can also be conceived as asymmetric units (Bashkirov, 1959).

Point symmetry versus space (or plane) symmetry. Point symmetry is often described as the symmetry of isolated shapes (crystal morphology) and their symmetry operations always leave at least one point unmoved. In contrast, space symmetry refers to the symmetry of lattices and translation appears now as an operation (in lattice vectors, glide planes/ lines and screw axes). The addition of translation to the 32 point-symmetry groups produces 230 possible space groups (Aroyo, 2016b). Similarly, in two dimensions there are only ten point groups and 17 plane groups.

Chirality. Chiral groups are those containing only symmetry operations of the first kind (i.e. operations that relate congruent objects) and having two enantiomorphic variants of the group. In contrast, achiral groups do not have variants and often contain operations of both the first and second kind (i.e. operations that relate enantiomorphous objects). For achiral groups containing operations of the second kind, two enantiomorphic versions of the asymmetric unit coexist within the unit cell.

\subsection{Models and suggested activities on periodic 2D lattices}

A set of several polygonal tiles is proposed as a way to experience the crystallographic restriction theorem. These are intended to let the student experience several configurations of periodic tilings trying to fill the plane without leaving gaps. The student can find that this is easily achievable using rectangular and regular three-, four- and six-sided polygons [Figs. $1(b), 1(c), 1(d)$ and $1(f)$ ], but that it is not possible using five-, seven- or eight-sided polygons [Figs. 1(e), 1(g) and 1(h)]. L-shaped tiles illustrate onefold rotational symmetry and it is worth noting that they also fill the plane without leaving gaps [Fig. 1(a)]. Besides finding the appropriate configurations to fill the space, other exercises that can be done with these tiles
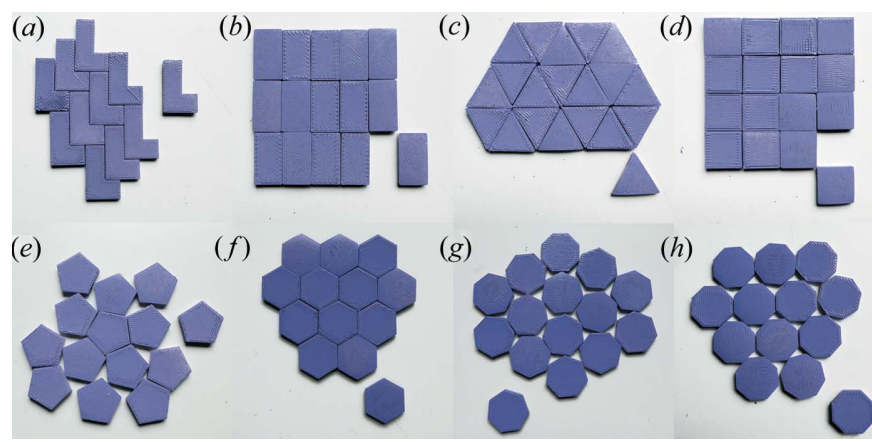

(g)

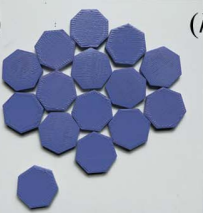

(h)

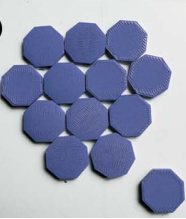

Figure 1

3D-printed tiles of several polygons with one- to eightfold rotational symmetry and configurations trying to fill the plane without gaps. (a) L-shaped tiles with onefold symmetry; $(b)$ rectangular tiles with twofold symmetry; $(c)$ equilateral triangular tiles with threefold symmetry; $(d)$ square tiles with fourfold symmetry; $(e)$ pentagonal tiles with fivefold symmetry; $(f)$ hexagonal tiles with sixfold symmetry; $(g)$ heptagonal tiles with sevenfold symmetry; $(h)$ octagonal tiles with eightfold symmetry. include the determination of the unit cell, the asymmetric unit and the corresponding plane group. Again, the tile with the lowest symmetry is particularly interesting because the unit cell is not so obvious and, as in this case the unit cell contains a single asymmetric unit, it is also interesting to check that the area of the tile constitutes the asymmetric unit as well as the unit cell (Fig. 2).

However, practice on plane symmetry determination can be performed in many other ways. There exist interactive tools to draw symmetry patterns with the desired plane-group symmetry (Eck, 2016). Also, decorative motifs in Moorish tiles and woodwork (Grünbaum et al., 1986; Aboufadil et al., 2013), Persian-style carpets (Bier, 1992; Atanassova \& Vassileva, 2017) and particularly the beautiful artworks by Escher (Bool et al., 1992) can be used to identify equivalent lattice points, symmetry elements, the unit cell, and ultimately the point and plane symmetry (Aroyo, 2016a). All these can also be adapted to $3 \mathrm{D}$-printed tiles that allow the construction of the symmetry pattern itself besides analysing the corresponding symmetry. As an example, an Escher-inspired lizard has been turned into a printable tile. The student could enjoyably practice with

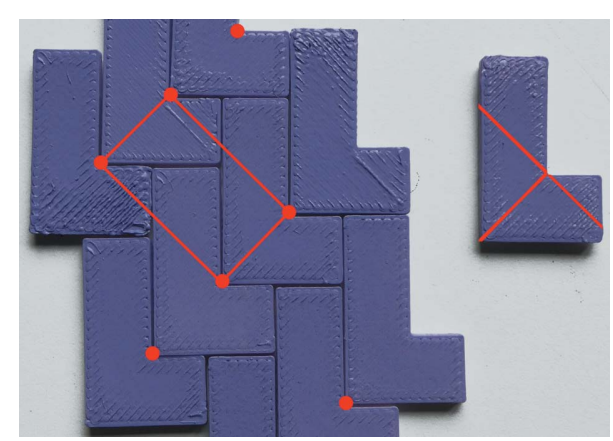

Figure 2

Detail of the L-shaped tiling. A set of equivalent points have been highlighted in red and a possible primitive unit cell has been drawn; the bits of tiles within a cell form a complete L-shaped tile.
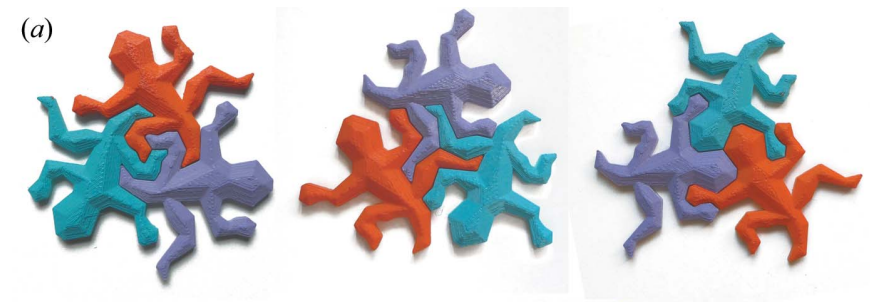

(b)

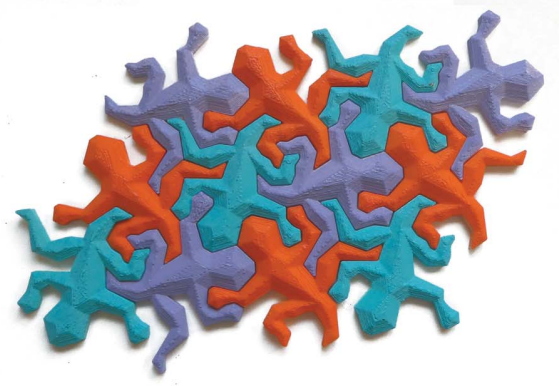

Figure 3

3D-printed Escher lizards. (a) Three identical lizards assembled in three different ways to highlight the three types of threefold rotational points;

(b) the lizards can tile the plane without leaving empty gaps. 


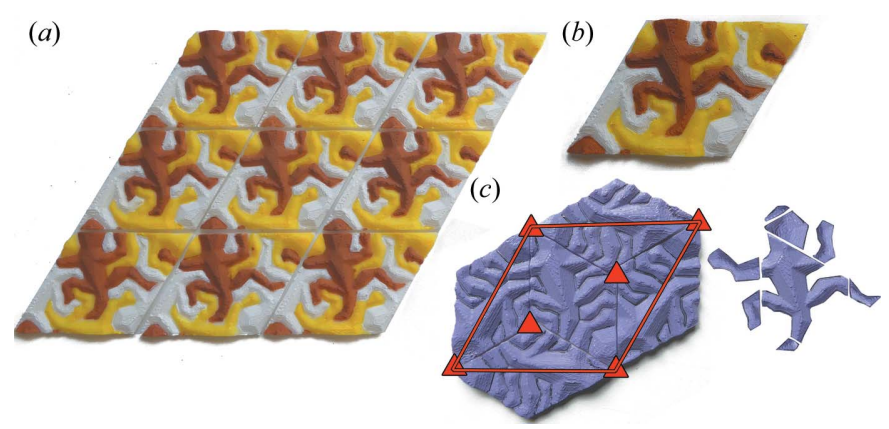

Figure 4

3D-printed hexagonal unit cells containing Escher lizards. (a) By translating the unit cells on the plane it is possible to tile it without leaving empty gaps. (b) Within a unit cell there are exactly three lizards. (c) By rotating and translating the asymmetric unit tiles it is also possible to tile the plane and to form the unit cell. Within a unit cell there is a full asymmetric unit and four halves of it. The unit-cell limits and its symmetry are indicated (left); the asymmetric unit contains exactly the parts to form a single lizard (right).

them. It can be quickly noted that three different orientations of the lizard suffice to fill the plane describing a periodic arrangement [Fig. 3(b)]. A deeper analysis could include the finding of three different threefold rotational centres [Fig. 3(a)] and the corresponding plane group (p3). Additionally, a tile corresponding to the unit cell has been designed [Fig. 4(b)]. These can also be used to build the same symmetry pattern [Fig. 4(a)] and to verify that the unit cell contains three lizards. Finally, tiles of the corresponding asymmetric unit have been designed, and these can be printed to build a single unit cell [Fig. 4(c)]. The student could note that all asymmetric units required to fill the unit cell are identical (the cell contains asymmetric units of only one hand) as the group (p3) is a purely rotational group where all symmetry operations are of the first kind. However, the group is achiral because a tiling using a mirrored version of the lizard would produce the very same $p 3$ plane group. The number of asymmetric units required to fill the cell is three, indicating that this is the order of the group. Also, it can be checked that an asymmetric unit contains the required parts to build exactly a single lizard [Fig. 4(c)]. In fact, it could be noted to the student that, conventionally, the limits of unit cells and asymmetric units are flat but the only true constraints regarding flat surfaces only affect asymmetric units where faces coincide with planes of mirror symmetry. Therefore, any of the three-lizard composites shown in Fig. 3(a) could perfectly act as unit cells and a single lizard can also be considered a possible asymmetric unit.

\subsection{Periodic 3D lattices}

2.3.1. Theoretical background. Besides the theoretical concepts mentioned previously (Section 2.1), two more concepts are relevant to the models representing periodic 3D lattices.

Holohedries. Despite the complexity of 3D space groups, the corresponding lattices built as a grid of translationequivalent points can be classified into just 14 types (Bravais lattices) (Aroyo et al., 2004) and there are 14 corresponding types of unit cells. Some of these cells contain exactly a single point of the Bravais lattice and they are called primitive cells. In contrast, other cells are larger and contain several lattice points (up to four for the $F$, or face-centred, cubic cell, $c F$ ). However, regardless of the primitive or non-primitive nature of the Bravais cells, they always exhibit the highest symmetry of their corresponding crystal system (holohedry) and obviously, as they are always parallelepipeds, they can be stacked in three dimensions, filling all the space without gaps or overlap.

Relation between rhombohedral $P$ and hexagonal $R$ cells. Also in the context of Bravais cells and lattices, the relation and transformation of the coordinate system between rhombohedral and hexagonal lattices within groups of the trigonal system is one of the subjects that is often perceived as confusing by students, in part for historical reasons (Nespolo $e t$ $a l ., 2018)$. The hexagonal crystal family $(h)$ includes both the rhombohedral and hexagonal lattice systems and the groups of their corresponding crystal systems (trigonal and hexagonal). Some space groups of the trigonal crystal system are conventionally described in a hexagonal lattice (actually two different hexagonal settings are possible) and others in a rhombohedral lattice, but all of them could be described using any of both lattice types. Therefore, for rhombohedral lattices, there is an equivalence between the primitive $(P)$ rhombohedral unit cell and a non-primitive $(R)$ hexagonal cell (Giacovazzo, 1992).

2.3.2. Models and suggested activities on periodic 3D lattices. It would be trivial to 3D-print parallelepipeds to experiment with assemblies of unit cells to check that they indeed fill the space. Anyone interested could download the STL files featuring illustrative examples of unit cells (for each lattice type), disregarding whether they are primitive or not, provided by Casas \& Estop (2015). However, readers should note that in the mentioned reference a hexagonal prism (comprising a composition of three different hexagonal unit cells) exemplifies the hexagonal lattice instead of the corresponding single unit cell. These models can also be used to check that their point symmetry matches that of holohedral point groups (Hahn et al., 2016).

Within groups of the trigonal system, the relation between rhombohedral $(P)$ and hexagonal $(R)$ cells is often illustrated using an image with their corresponding axonometric projections [see for instance Fig. 5.1.3.6 of Arnold (2005) or Fig. 1.17 of Giacovazzo (1992)]. 3D printing can contribute to developing understanding of such a relation, allowing the student to build hexagonal cells using rhombohedral cells. A dissection puzzle consisting of a central single primitive rhombohedral cell along with 32 bits of rhombohedral cells conveniently cut has been designed [Fig. 5(a)], with the vertices corresponding to lattice points painted in black. The pieces can be easily stuck using Blu-Tack or a similar product. Within the supporting information, along with all the printable files, the interested reader can find in Fig. S1 the different steps to assemble the whole puzzle that forms four non-primitive $R$ hexagonal cells [Fig. 5(b)]. These $R$ cells contain two extra lattice points besides the one at $(0,0,0)$ - they are located at 
$(2 / 3,1 / 3,1 / 3)$ and $(1 / 3,2 / 3,2 / 3)$ and can be seen by partially disassembling the puzzle [Fig. 5(c)].

Besides solid unit cells defined by lattice points, 3D printing can also be used to produce unit cells filled with their atomic contents (Rodenbough et al., 2015). The addition of the atomic contents allows the description of any of the 230 crystallographic point groups. One of the technical issues in the production of such models is tuning the way the atoms are bonded to each other and whether the limits of the cell itself need to be printed. A low quantity of supports and attachment points produces cleaner but also weaker models. However, a model of a whole unit cell has low versatility for playing with operations, elements and other symmetry concepts. For that matter, a model of the asymmetric unit would be much more
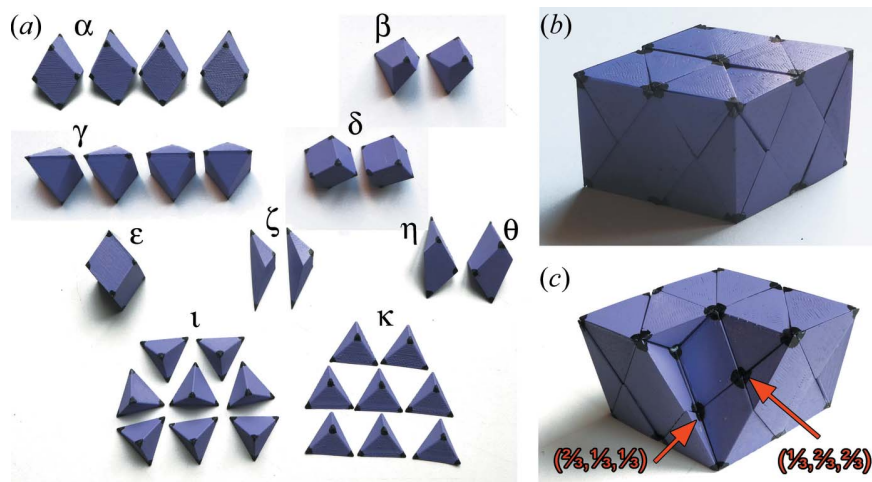

Figure 5

3D-printed dissection puzzle of a set of four $R$ hexagonal cells. (a) The 33 required pieces grouped and labelled by forms; $(b)$ the assembled puzzle; (c) some pieces have been removed from the assembled puzzle to show the two extra lattice points of one of the $R$ cells.
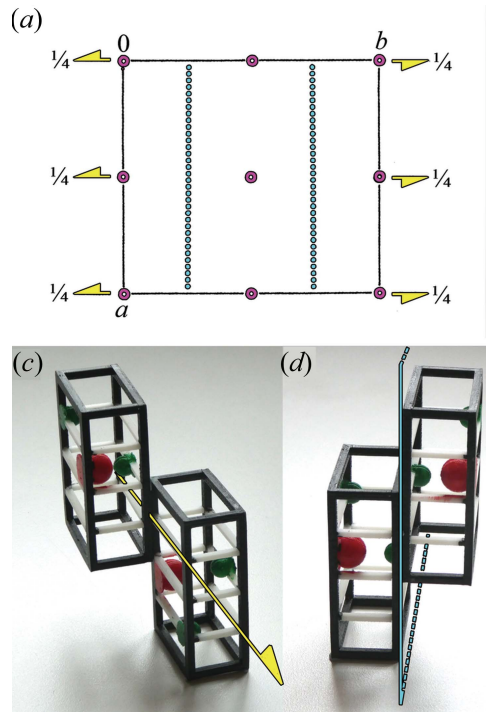

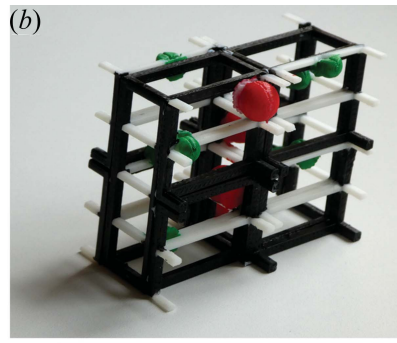

(e)

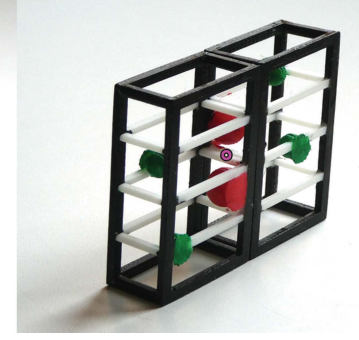

Figure 6

3D-printed unit cell and asymmetric units of space group $P 2_{1} / c$. (a) Schematic representation of space group $P 2_{1} / c ;(b) 3 \mathrm{D}$-printed $P 2_{1} / c$ unit cell assembled using two full unit cells and eight quarters of a unit cell; $(c)$ two identical asymmetric units related by a twofold screw axis; $(d)$ two enantiomorphic unit cells related by a $c$ glide plane; (e) two enantiomorphic unit cells related by a centre of symmetry. useful. To illustrate the full potential of 3D-printed models of asymmetric units including atomic positions, a model of the monoclinic $P 2_{1} / c$ group has been chosen. This group is known to be the most frequent space group, among both organic (36\%) (Mighell et al., 1983) and inorganic (39\%) (Urusov \& Nadezhina, 2009) compounds. The printable model comprises two enantiomorphic asymmetric units and $\frac{1}{4}$ portions of them in such a way that the full unit cell can be assembled [Figs. 6( $a)$ and $6(b)]$. The student can be guided to understand that this implies that $P 2_{1} / c$ contains symmetry operations of the second kind (and therefore is necessarily achiral); another interesting consideration is that the limits of the unit cell do not necessarily correspond to the limits of the asymmetric unit.

The asymmetric unit has been designed to contain three atoms (two of one type and another of a different kind), and the atoms have been placed close to the external surface of the asymmetric unit in order to avoid the need for long supports to hold them in place. Besides the edges of the asymmetric unit, extra shafts parallel to the edges along $\mathbf{a}$ and $\mathbf{b}$ have been added at heights of $\frac{1}{4}, \frac{1}{2}$ and $\frac{3}{4}$ to give robustness to the model and to have a reference of the mentioned heights. The models can be used to visualize separately the symmetry elements of the $P 2_{1} / c$ group (a twofold screw axis, a $c$ glide plane and a centre of symmetry). This can be proposed to students as an exercise to confirm that screw axes only contain symmetry operations of the first kind and therefore two identical asymmetric units are required to illustrate the objects that relate this element [see Fig. 6(c)]. In contrast, both the glide plane and the centre of symmetry also contain symmetry operations of the second kind and relate enantiomorphic asymmetric units [see Figs. 6(d) and 6(e)].

\section{Aperiodic arrays}

\subsection{Aperiodic 2D tilings}

3.1.1. Theoretical background. Similarly to periodic lattices, aperiodic lattices (or rather arrays) corresponding to aperiodic crystals are better understood with 2D examples of aperiodic tilings. Non-periodic plane tessellation or tiling is a topic in geometry with applications in many scientific fields, including crystallography. The evolution of knowledge on aperiodic tilings shows a clear similarity with that related to quasicrystals. A 3D generalization of aperiodic tilings was proposed by Ammann (Senechal, 2004) and, soon after the discovery of quasicrystals, aperiodic tilings were considered possible models for quasicrystals.

Some of the relevant concepts that can be explained using the presented models of aperiodic tilings are commented on very briefly below.

Prototiles. A set of tiles (actually called prototiles) whose tilings are all non-periodic is called aperiodic. Such a set of prototiles was conjectured, in the mid-20th century, not to exist (Wang, 1961). However, in 1966, Berger found the first one consisting of 20426 prototiles (Berger, 1966) and, before long, many other sets were found with fewer tiles. In the early 1970s Penrose (Penrose, 1974) and Ammann (Ammann et al., 
1992) found sets with only two prototiles, thus differing only by one tile with respect to the periodic tiling of crystallographic plane groups. In fact, there is ongoing research to construct aperiodic tilings from a single prototile (Baake et al., 2012).

Matching rules. To force the non-periodicity of possible tilings, the prototiles have an additional structure usually in the form of deformations, decorated edges or a decorated surface. These are generally referred to as matching rules (Goodman-Strauss, 1998). These decorations should fit consistently to build a non-periodic array.

Inflation. Even respecting the matching rules, sometimes it is possible to end up with a configuration that cannot be infinitely extended without leaving gaps. To avoid this, an aperiodic tiling should be constructed in such a way that the pattern could be reproduced exactly at a larger scale by a composition process usually called 'inflation tiling'. Inflated tiles are patches of tiling built using prototiles (and occasionally half-prototiles) that reproduce the shape of the prototiles at a higher scale. Interestingly the inflation process could be infinitely repeated at larger and larger scales (and the inversion process, called deflation, as well). The scale ratio between a tiling and its inflated version is called the inflation factor.

Metallic ratios. Among the best-known examples of aperiodic tilings, Penrose tilings (Penrose, 1974) have an inflation factor of $\left(5^{1 / 2}+1\right) / 2$, which is known as the golden ratio $(\varphi$, or sometimes $\tau$ ). The symmetry of Penrose tilings contains fivefold symmetry. Interestingly the golden ratio $(\varphi)$ can also be found in the ratio of the chord length to the side length of a regular pentagon. Among the Ammann tilings (Grünbaum \& Shephard, 2016) there are two (A4 and A5) with an inflation factor of $2^{1 / 2}+1$, which is known as the silver ratio $\left(\delta_{\mathrm{S}}\right)$. The A5 tiling contains eightfold symmetry. Remarkably, the silver ratio $\left(\delta_{\mathrm{S}}\right)$ is found in a regular octagon as twice the ratio of the inradius to the side length.

Proof of aperiodicity. A self-similar array of tiles (i.e. one that can be infinitely inflated) can fill all the plane without leaving gaps. The resulting array is necessarily aperiodic (without translational symmetry), because once we establish the non-existence of translations in a patch that can be inflated, no translation will exist at any other scale.

3.1.2. Models and suggested activities on aperiodic 2D tilings. The construction of a bidimensional periodic lattice using appropriate tiles would be trivial for most students; in contrast, the construction of an aperiodic array is less obvious. Three two-prototile tilings are proposed [Fig. 7(a)] to provide the student with experience of aperiodic arrays: the P2 (kite and dart) and P3 (rhomb) tilings by Penrose and the A5 (Ammann-Beenker) tiling by Ammann (Grünbaum \& Shephard, 2016). One of these tilings (P2) has actually already starred in a recent publication on the use of Penrose tiles for teaching the aperiodicity concept (Rossi et al., 2020). It is worth repeating that only the set of prototiles whose tilings are all non-periodic is called aperiodic. Taking into account only the shape of the suggested tiles, it is actually possible to build periodic plane lattices and this could also be a suggested exercise for students, along with symmetry identification and plane group and unit-cell determination. For examples of periodic lattices using the $\mathrm{P} 2$ prototiles see the work of Rossi et al. (2020).

To assemble successfully a non-periodic tiling with the presented prototiles, the student can be prompted to follow decorations and inflation. (i) Taking advantage of the possibilities of 3D printing, the known original decorations of these tilings that force aperiodicity have been designed as relief decorations; therefore the student has to place the tiles in such a way that the decorations must always be connected between neighbouring tiles. (ii) As a further guide, the student must follow a pattern that could be inflated [Fig. 7(e)]. Actually, a first exercise with the models could be the construction of single inflated tiles [Figs. 7(b), 7(c) and 7(d)] and verification that the inflation factor (i.e. the scaling factor relating a

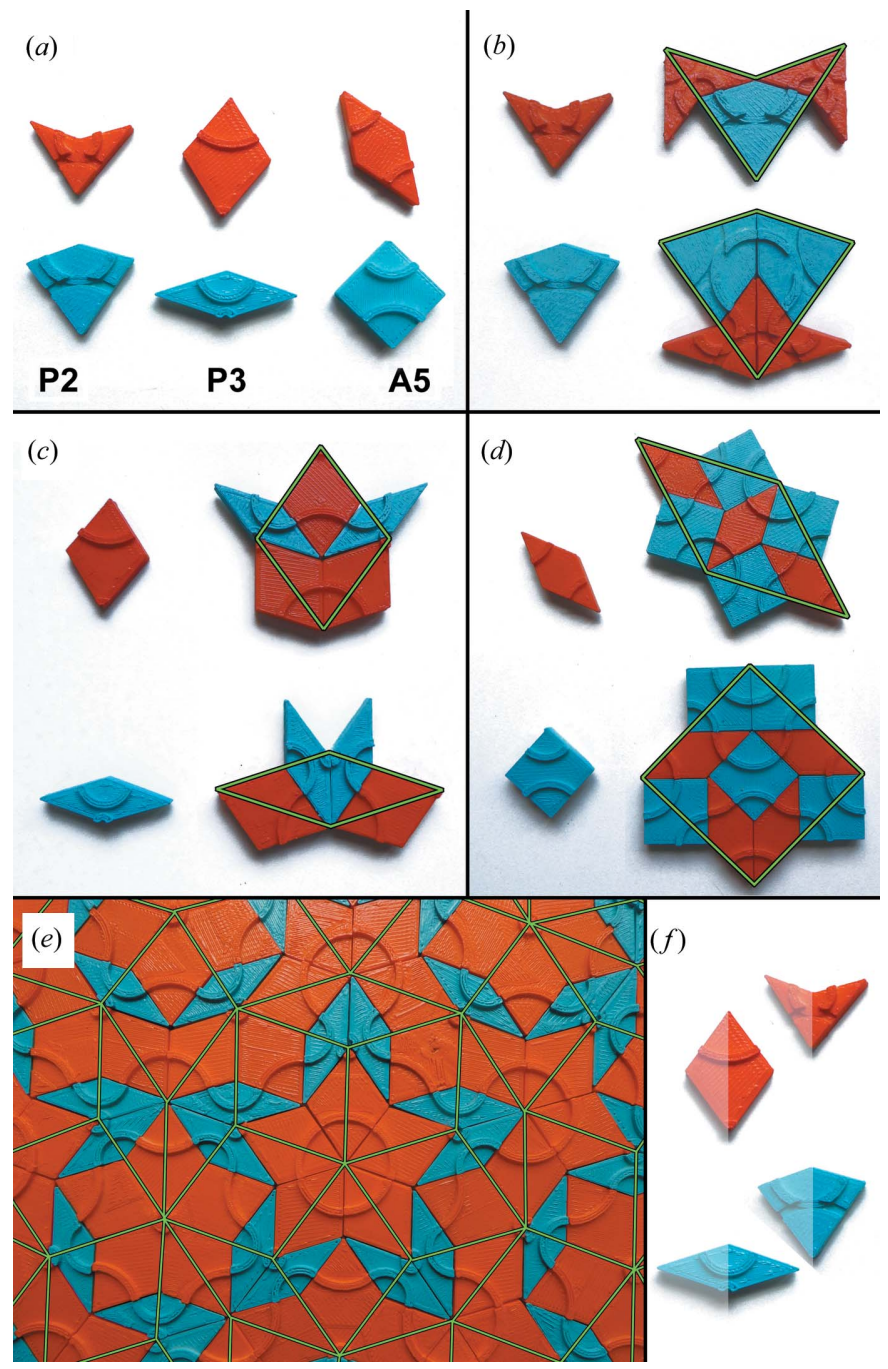

Figure 7

3D-printed prototiles for aperiodic tilings. (a) The pair of prototiles for P2, P3 and A5 aperiodic tilings; $(b)$ the P2 prototiles and their inflated version; $(c)$ the $\mathrm{P} 3$ prototiles and their inflated version; $(d)$ the A5 prototiles and their inflated version; (e) P3 aperiodic tiling with the same tiling at a higher scale (using the inflated prototiles) drawn on it; $(f)$ bisected $\mathrm{P} 2$ and $\mathrm{P} 3$ prototiles showing identical obtuse (orange) and acute (light blue) Robinson triangles. 
prototile to its inflated version) is $\varphi$ [the golden ratio, $\left.\left(5^{1 / 2}+1\right) / 2\right]$ for $\mathrm{P} 2$ and $\mathrm{P} 3$ tilings and $\delta_{\mathrm{S}}$ (the silver ratio, $\left.2^{1 / 2}+1\right)$ for the A5 tiling. The notion of inflation, the possibility of repeating the process at larger scales, can be used to discuss self-similarity and to use this as a proof of the inexistence of translational symmetry.

Additional exercises can be proposed using the similar P2 and P3 tilings. Both Penrose tilings can be bisected to form golden triangles and gnomons, which sometimes are called acute and obtuse Robinson triangles, respectively [Fig. 7(f)]. The student could verify that these isosceles triangles are truly golden as the ratio of the duplicated side length to the base is indeed the golden ratio (for the acute triangle) or its reciprocal (for the obtuse triangle) (Loeb, 1989).

Finally, once a large area has been tiled following the appropriate rules, the student could try to find the plane symmetry of them, in particular noting the absence of any translational symmetry. P2 and P3 tilings have exactly the same symmetry consisting of a single fivefold rotation point and five reflection lines and many points with local pentagonal symmetry [Figs. $8(a)$ and $8(b)$ ]. In contrast, the A5 tiling, formed by squares and $45^{\circ}$ rhombuses (lozenges), has a single eightfold rotation point and eight reflection lines, and many points with local octagonal symmetry [Fig. 8(c)].

To sum up, the possibility of assembling the tilings from 3Dprinted decorated pieces allows the students to experience many related concepts, including rotation points, reflection lines, local symmetries, inflation, inflation factors, metallic
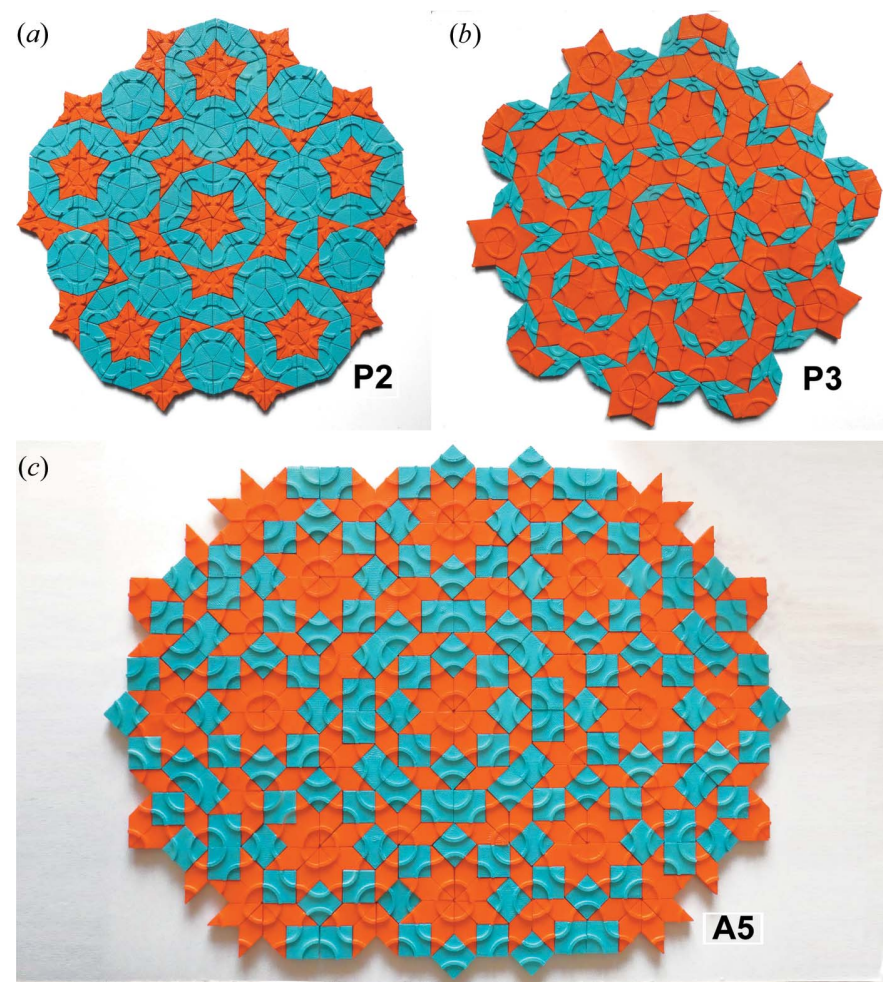

Figure 8

3D-printed aperiodic tilings. (a) P2 tiling with pentagonal symmetry; (b) P3 tiling with pentagonal symmetry; (c) A5 tiling with octagonal symmetry. ratios etc. Additionally, empirical measurements could be done to verify the appearance of golden and silver ratios as well as their values.

\subsection{Aperiodic $3 \mathrm{D}$ arrays}

3.2.1. Theoretical background. The mathematical description of aperiodic crystals is often carried out considering atoms as multidimensional atomic surfaces arranged periodically in a superspace; the projection in a $3 \mathrm{D}$ space results in an aperiodic arrangement of atoms (Yamamoto, 1996). There are alternative descriptions of aperiodic crystals, for instance a model based on quasi-unit cells (Steinhardt et al., 1998) that overlap each other or a statistical method based on the concept of average unit cell (Strzalka et al., 2016). These methods are complicated for undergraduate students and they would not be advisable as a first introduction to aperiodic crystals. Nevertheless, some theoretical concepts should be mentioned in order to understand and take full advantage of the presented models of aperiodic crystals.

Icosahedral tiling. Analogously to the 2D tilings, it is possible to produce a 3D aperiodic tiling using a number of polyhedra that act as prototiles in a sort of 3D Penrose tiling known as an icosahedral tiling. The resulting structure exhibits icosahedral point-group symmetry (Litvin, 1991), a symmetry found in several aperiodic crystals exhibiting fivefold rotation axes such as the triacontahedron, the icosahedron or the pentagonal dodecahedron (Zupaniè et al., 2011; Canfield et al., 2010). Interestingly, these morphologies were reported by Romé de L'Isle in the 18th century (Pina \& López-Acevedo, 2016).

Golden rhombohedra. The simplest icosahedral tilings employ only two rhombohedric prototiles, known as the golden or Ammann rhombohedra (Kramer \& Neri, 1984). In fact, Ammann was the first to suggest that these polyhedra could tile space aperiodically (Senechal, 2004), although these rhombohedra had already been described much earlier by Kowalewski (1938). Golden rhombohedra are rhombohedra whose faces consist of congruent golden rhombuses [i.e. rhombuses whose diagonals are in the golden ratio $(\varphi)$ ]. For one of the golden rhombohedra three obtuse angles meet at the two polar axis vertices (oblate), and for the other three acute angles meet at the two polar axis vertices (prolate). A theoretical approach to generate 3D icosahedral tilings with such golden rhombohedra has been undertaken by several authors (Kramer \& Neri, 1984; Ogawa, 1985; Levine \& Steinhardt, 1986; Socolar \& Steinhardt, 1986) using different methods. However, such an aperiodic array using only two prototiles would require an intricate selection of proper matching rules in the form of decorations that are very difficult to state (Hann et al., 2016; Lifshitz, 2011).

Cut-and-project method. Among the different methods to obtain or describe aperiodic arrays, the cut-and-project method is particularly common. This method produces the aperiodic array from an irrational 3D slice through a higherdimensional periodic lattice (Kramer \& Neri, 1984; Lord, 1991). For example, using the cut-and-project method, the 
Penrose tiling can be obtained by cutting a $2 \mathrm{D}$ slice through a 4D hyperrhombohedral lattice and the icosahedral tiling by cutting a 3D slice through a 6D hypercubic lattice (Steurer \& Haibach, 1999).

Golden isozonohedra. Among zonohedra (i.e. convex polyhedra whose faces are centrally symmetric), golden isozonohedra are those whose faces are golden rhombuses. There exist only five golden isozonohedra: the oblate and prolate golden rhombohedra, the triacontahedron, the rhombic dodecahedron, and the rhombic icosahedron. Socolar \& Steinhardt (1986) proposed the construction of the icosahedral tiling using four golden isozonohedra (all but the oblate rhombohedron) as a set of four prototiles. This tiling keeps the icosahedral symmetry in the $3 \mathrm{D}$ tiling.

3.2.2. Models and suggested activities on aperiodic 3D crystals. The accurate mathematical description of aperiodic crystals is intricate. Although schematic diagrams can be used to interpret the cut-and-project method for generating an aperiodic unidimensional array from a 2D periodic lattice (Fang et al., 2017), a hands-on learning approach using convenient tools could be a better way to approach this complicated subject. On the other hand, the construction of a 3D model of an aperiodic crystal is possibly a more attractive and challenging activity than building plane aperiodic tilings.

The 3D printing of many copies of the two golden rhombohedra (Fig. 9, inset) will produce the pieces to allow many worthwhile exercises connected to icosahedral tilings. To start with, the student could examine the geometry of printed golden rhombohedra to verify that all the faces of both are identical golden rhombuses, i.e. their diagonals are in the

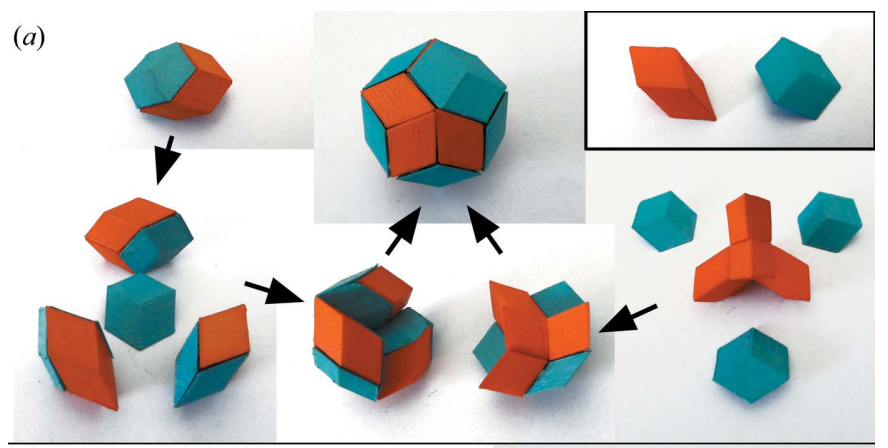

(b)

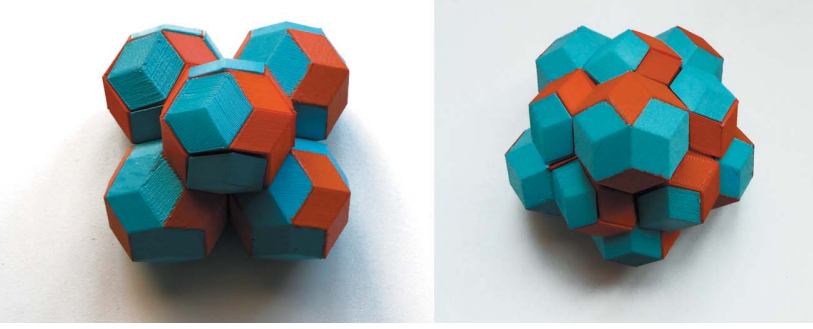

Figure 9

(a) Steps showing how to assemble a rhombic triacontahedron using ten oblate and ten prolate 3D-printed golden rhombohedra. Inset: the oblate (light blue) and prolate (orange) golden rhombohedra. (b) The triacontahedra can form 3D ensembles filling the empty spaces with golden rhombohedra. golden ratio $(\varphi)$ and the volume ratio between the prolate and oblate rhombohedra is also $\varphi$.

The next step would be to try to use the golden rhombohedra to assemble a body containing fivefold symmetry and without leaving empty spaces. Twenty rhombohedra (ten oblate and ten prolate) can be assembled to form a rhombic triacontahedron [see Fig. $9(a)$ ] as already shown by Kowalewski (1938). Then, several triacontahedra can be assembled, for instance sharing faces, leaving spaces that can be completely filled with oblate and prolate rhombohedra [Fig. 9(b)]. However, it is easy to find combinations where the characteristic pentagonal symmetry is lost and it is actually impossible to keep the ideal icosahedral symmetry considering only the golden rhombohedra as prototiles (Madison \& Madison, 2019).

Another step would be to use the golden rhombohedra to assemble the other golden isozonohedra besides the triacontahedron (and the golden rhombohedra for themselves). These are the rhombic dodecahedron and the rhombic icosahedron. The first comprises two prolate and two oblate rhombohedra, and the latter five prolate and five oblate rhombohedra. Once assembled, another suggested exercise for students could be to determine the point-group symmetry of the five isozonohedra [Fig. 10(a)]. In particular it should be noted that the rhombic icosahedron contains a single fivefold symmetry axis (point symmetry would correspond to group $\overline{5} m$ ) and the triacontahedron contains six fivefold symmetry axes (point symmetry would correspond to group $\overline{53} \mathrm{~m}$ ). The construction of the four prototiles (i.e. all isozonohedra but the oblate rhombohedron) suggested by Socolar \& Steinhardt
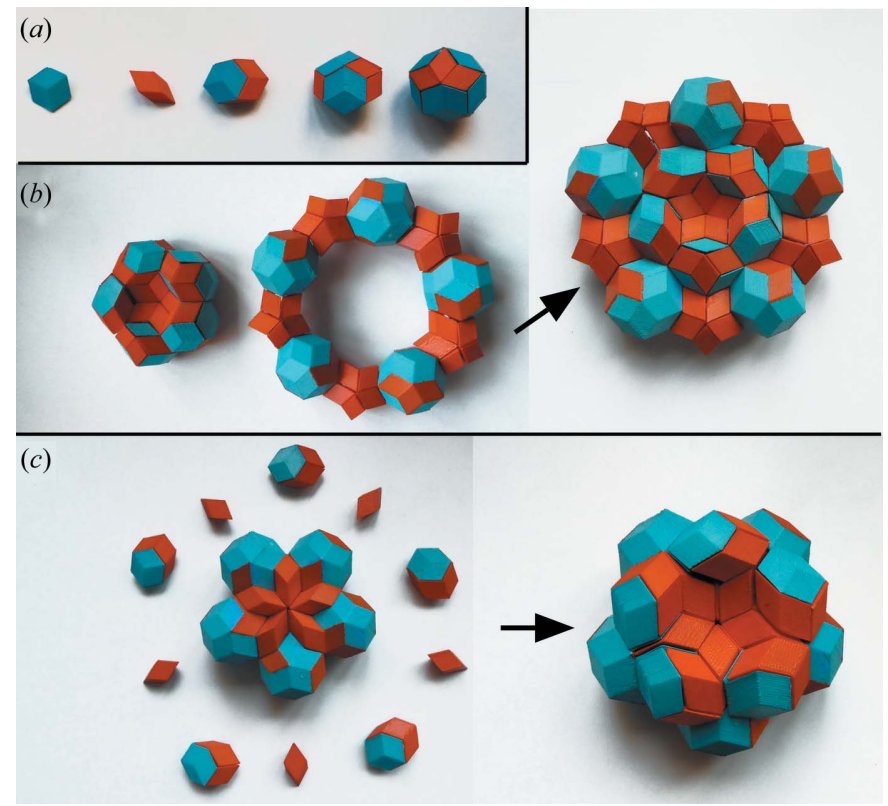

Figure 10

(a) The five golden isozonohedra: from left to right, oblate rhombohedron, prolate rhombohedron, rhombic dodecahedron, rhombic icosahedron and triacontahedron. The last three can be assembled combining the two golden rhombohedra. (b), (c) Two examples of tilings exhibiting a topography with fivefold symmetry using assemblies of 3D-printed golden rhombohedra previously assembled as zonohedra. 


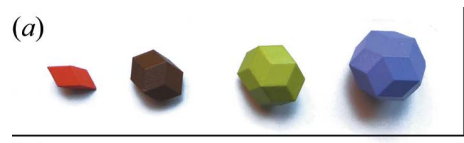

(b)
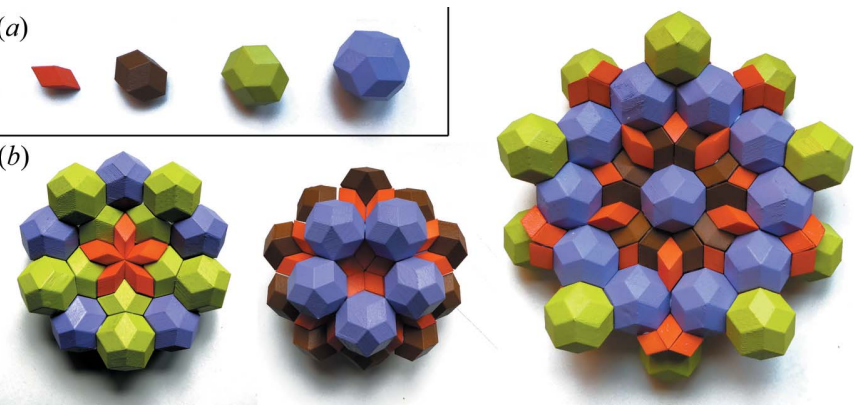

Figure 11

(a) 3D printings of the four types of zonohedra used as prototiles in the icosahedral tiling painted in different colours. (b) Three examples of tilings using the zonohedra that exhibit a fivefold symmetry axis.

(1986) could be a good starting point to attempt the construction of an aperiodic array keeping the icosahedral symmetry (Fig. 10). Using pre-assembled isozonohedra the student could find it easier to build assemblages of golden rhombohedra which define a surface topography that holds the fivefold symmetry axes [Figs. $10(b)$ and $10(c)$ ]. Also, the student could try to verify that in such icosahedral tilings the frequency of appearance of the prolate rhombohedron is $\varphi$ times higher than that of the oblate rhombohedron.

Finally, a more elaborate assemblage could be done by painting in a different colour every type of zonohedron [Fig. 11(a)]. In this way, it is possible to create a tiling that keeps the icosahedral symmetry including both the surface topography and the colour distribution. Some suggested exercises could be to build the inflated zonohedra as described and beautifully illustrated in the work of Madison (2015a,b), as well as self-similar layers and slices perpendicular to the fivefold axis (Madison, 2015a; Madison \& Madison, 2019); examples are given in Fig. 11(b). The inflation factor of these self-similar layers is $\varphi^{3}$ (Katz \& Duneau, 1986).

\section{Conclusion}

Different 3D-printed designs have been presented to apply hands-on learning to a number of concepts usually taught in basic crystallography courses using both $2 \mathrm{D}$ and $3 \mathrm{D}$ examples: e.g. periodicity, the crystallographic restriction theorem, unit cell, point symmetry, asymmetric unit, chirality, lattices and symmetry elements. A similar approach has been undertaken to introduce a number of notions that concern aperiodic crystals and that, commonly, are not taught due to their difficulty both conceptually and from a mathematical point of view. These include non-periodicity, aperiodicity, self-similarity, proof of the absence of translational symmetry and the potentially infinite size of aperiodic media.

The presented and discussed materials comprise the following. (i) Several designs intended for the study of periodic media: eight types of polygonal tiles and an Escherinspired lizard tile (including the single lizard and a corresponding unit cell and asymmetric unit), a dissection puzzle of four hexagonal cells including ten types of pieces, a model of the unit cell (bearing atoms) of a monoclinic space group containing ten pieces. (ii) Other designs intended for the study of aperiodic media: three pairs of prototiles for aperiodic 2D tilings and five polyhedra for 3D tilings. All these designs are made freely available as printable STL files that can be found in the supporting information. The printed materials and their related examples should provide students and teachers with additional resources to overcome the difficulties of learning and teaching concepts of both periodic and aperiodic crystals. Educators are encouraged to use and adapt these models in their lectures.

\section{References}

Aboufadil, Y., Thalal, A. \& Raghni, M. A. E. I. (2013). J. Appl. Cryst. 46, 1834-1841.

Ammann, R., Grünbaum, B. \& Shephard, G. C. (1992). Discrete Comput. Geom. 8, 1-25.

Arnold, H. (2005). International Tables for Crystallography, Vol. A, Space-Group Symmetry, edited by T. Hahn, ch. 5.1, pp. 78-85. Dordrecht: Springer.

Aroyo, M. I. (2016a). Editor. International Tables for Crystallography, Vol. A, Space-Group Symmetry, ch. 2.2, pp. 175-192. Chichester: Wiley.

Aroyo, M. I. (2016b). Editor. International Tables for Crystallography, Vol. A, Space-Group Symmetry, ch. 2.3, pp. 193-687. Chichester: Wiley.

Aroyo, M. I., Müller, U. \& Wondratschek, H. (2004). International Tables for Crystallography, Vol. A1, Symmetry Relations Between Space Groups, edited by H. Wondratschek \& U. Müller, ch. 1.1, pp. 2-5. Dordrecht: Springer Netherlands.

Arribas, V., Casas, L., Estop, E. \& Labrador, M. (2014). Comput. Geosci. 62, 53-61.

Atanassova, R. \& Vassileva, R. (2017). Geol. Balc. 46, 27-31.

Authier, A. \& Chapuis, G. (2017). A Little Dictionary of Crystallography. Chester: IUCr.

Baake, M., Gähler, F. \& Grimm, U. (2012). Symmetry (Basel), 4, 581602.

Bashkirov, N. M. (1959). Sov. Phys. Crystallogr. pp. 442-447.

Berger, R. (1966). Mem. Am. Math. Soc. 66, 1-72.

Bier, C. (1992). Text. Mus. J. 31, 53-70.

Bool, F. H., Kist, J. R., Wierda, F. \& Locher, J. L. (1992). M. C. Escher - His Life and Complete Graphic Work. New York: Harry N. Abrams.

Borchardt-Ott, W. (2012). Crystallography, an Introduction. Heidelberg: Springer.

Canfield, P. C., Caudle, M. L., Ho, C.-S., Kreyssig, A., Nandi, S., Kim, M. G., Lin, X., Kracher, A., Dennis, K. W., McCallum, R. W. \& Goldman, A. I. (2010). Phys. Rev. B, 81, 020201.

Casas, L. \& Estop, E. (2015). J. Chem. Educ. 92, 1338-1343.

Chen, T.-H., Lee, S., Flood, A. H. \& Miljanić, O. S. (2014). CrystEngComm, 16, 5488-5493.

Coxeter, H. S. M. (1973). Regular Polytopes. New York: Dover Publications.

Crundwell, G., Phan, J. \& Kantardjieff, K. A. (1999). J. Chem. Educ. 76, 1242.

Eck, D. J. (2016). Wallpaper Symmetry, http://math.hws.edu/eck/js/ symmetry/wallpaper.html

Fang, F., Hammock, D. \& Irwin, K. (2017). Crystals, 7, 304.

Fanwick, P. E. (2007). Annual Reports in Computational Chemistry, Vol. 3, edited by D. C. Spellmeyer \& R. Wheeler, pp. 85-98. Amsterdam: Elsevier.

Fisher, I. R., Cheon, K. O., Panchula, A. F., Canfield, P. C., Chernikov, M., Ott, H. R. \& Dennis, K. (1999). Phys. Rev. B, 59, 308-321.

Giacovazzo, G. (1992). Editor. Fundamentals of Crystallography, pp. 19-22. Oxford: IUCr/Oxford University Press.

Goodman-Strauss, C. (1998). Ann. Math. 147, 181-223. 
Gražulis, S., Sarjeant, A. A., Moeck, P., Stone-Sundberg, J., Snyder, T. J., Kaminsky, W., Oliver, A. G., Stern, C. L., Dawe, L. N., Rychkov, D. A., Losev, E. A., Boldyreva, E. V., Tanski, J. M., Bernstein, J., Rabeh, W. M. \& Kantardjieff, K. A. (2015). J. Appl. Cryst. 48, 1964-1975.

Grimm, U. (2015). Acta Cryst. B71, 258-274.

Grünbaum, B., Grünbaum, Z. \& Shepard, G. C. (1986). Comput. Math. Appl. 12, 641-653.

Grünbaum, B. \& Shephard, G. C. (2016). Tilings and Patterns. New York: Dover Publications.

Hahn, T., Klapper, H., Müller, U. \& Aroyo, M. I. (2016). International Tables for Crystallography, Vol. A, Space-Group Symmetry, edited by M. I. Aroyo, ch. 3.2, p. 720. Chichester: Wiley.

Hann, C. T., Socolar, J. E. S. \& Steinhardt, P. J. (2016). Phys. Rev. B, 94, 014113.

Hluchy, M. M. (1999). J. Geosci. Educ. 47, 236-240.

Janssen, T., Chapuis, G. \& de Boissieu, M. (2018). Aperiodic Crystals: From Modulated Phases to Quasicrystals: Structure and Properties. Oxford University Press.

Katz, A. \& Duneau, M. (1986). J. Phys. Fr. 47, 181-196.

Kortan, A. R., Chen, H. S., Parsey, J. M. \& Kimerling, L. C. (1989). J. Mater. Sci. 24, 1999-2005.

Kowalewski, G. (1938). Der Keplersche Körper und andere Bauspiele. Leipzig: K. F. Koehlers Antiquarium.

Kramer, P. \& Neri, R. (1984). Acta Cryst. A40, 580-587.

Levine, D. \& Steinhardt, P. J. (1986). Phys. Rev. B, 34, 596-616.

Lifshitz, R. (2011). Isr. J. Chem. 51, 1156-1167.

Litvin, D. B. (1991). Acta Cryst. A47, 70-73.

Loeb, A. L. (1989). Comput. Math. Appl. 17, 33-48.

Lord, E. A. (1991). Curr. Sci. 61, 313-319.

Maciá Barber, E. (2019). Appl. Sci. 9, 2132.

Macrae, C. F., Sovago, I., Cottrell, S. J., Galek, P. T. A., McCabe, P., Pidcock, E., Platings, M., Shields, G. P., Stevens, J. S., Towler, M. \& Wood, P. A. (2020). J. Appl. Cryst. 53, 226-235.

Madison, A. E. (2015a). RSC Adv. 5, 79279-79297.
Madison, A. E. (2015b). RSC Adv. 5, 5745-5753.

Madison, A. E. \& Madison, P. A. (2019). Proc. R. Soc. A Math. Phys. Eng. Sci. 475, 20180667.

Mighell, A. D., Himes, V. L. \& Rodgers, J. R. (1983). Acta Cryst. A39, 737-740.

Nespolo, M., Aroyo, M. I. \& Souvignier, B. (2018). J. Appl. Cryst. 51, $1481-1491$.

Ogawa, T. (1985). J. Phys. Soc. Jpn, 54, 3205-3208.

Penrose, R. (1974). Bull. Inst. Math. Its Appl. pp. 266-271.

Pernaa, J. \& Wiedmer, S. (2019). Chem. Teach. Int. 20190005.

Pett, V. B. (2010). J. Appl. Cryst. 43, 1139-1143.

Pina, C. \& López-Acevedo, V. (2016). Crystals, 6, 137.

Rodenbough, P. P., Vanti, W. B. \& Chan, S.-W. (2015). J. Chem. Educ. 92, 1960-1962.

Rossi, S., Rossi, C. \& Accorigi, N. (2020). J. Chem. Educ. 97, 13911395.

Scherrer, W. (1946). Elem. Math. 1, 97-98.

Senechal, M. (2004). Math. Intelligencer, 26, 10-21.

Senechal, M. (2015). Acta Cryst. B71, 250-251.

Sharma, B. D. (1983). J. Chem. Educ. 60, 462.

Shechtman, D., Blech, I., Gratias, D. \& Cahn, J. W. (1984). Phys. Rev. Lett. 53, 1951-1953.

Socolar, J. E. S. \& Steinhardt, P. J. (1986). Phys. Rev. B, 34, 617-647.

Steinhardt, P. J., Jeong, H.-C., Saitoh, K., Tanaka, M., Abe, E. \& Tsai, A. P. (1998). Nature, 396, 55-57.

Steurer, W. \& Haibach, T. (1999). Acta Cryst. A55, 48-57.

Strzalka, R., Buganski, I. \& Wolny, J. (2016). Crystals, 6, 104.

Tsai, A.-P. \& Cui, C. (2015). Handbook of Crystal Growth, edited by T. Nishinaga, pp. 1113-1156. Boston: Elsevier.

Urusov, V. S. \& Nadezhina, T. N. (2009). J. Struct. Chem. 50, 22-37.

Wang, H. (1961). Bell Syst. Tech. J. 40, 1-41.

Yamamoto, A. (1996). Acta Cryst. A52, 509-560.

Zupaniè, F., Bonèina, T. \& Rozman, N. (2011). Quasicrystals: Types, Systems and Techniques, edited by B. E. Puckermann, pp. 195-217. New York: Nova Science Publishers. 